\title{
FULLY AUTOMATIC MIXING MACHINE
}

MixStar-eMotion is DMG UK's latest generation fully automatic mixing machine designed for use with their market leading MixStar impression materials - Honigum-MixStar, Silagum-MixStar and StatusBlue.

The MixStar-eMotion possesses three different, electronically controlled speed settings that ensure consistent results even with more viscous materials. The top speed enables dispensing at twice the speed of its predecessor. Setting and working time in the mouth can be adjusted with two timers and saved in programmes. The mixing process can be started with a simple press of a button or by using an optional footswitch, which can be plugged in for hands free operation. With the aid of a serial interface, software can be updated any time via the Internet.

The MixStar-eMotion mixing unit is a fully automatic mixing machine designed to deliver perfectly mixed, homogenous, bubble free impression material directly into the impression tray or syringe. Extremely userfriendly, it is easy to operate at the touch of a button, enabling both of the operator's hands to remain free to hold the tray or syringe during filling. It offers user-friendly mixing and dispensing speeds plus quick and easy cartridge and mixing tip exchange when required.

Used in combination with DMG's MixStar range of impression materials it helps save valuable surgery time, waste and mess. Reader response number 56

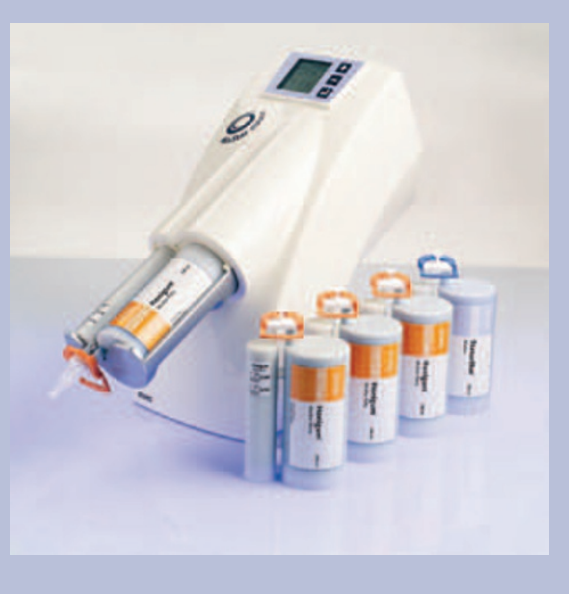

\section{BE A FIRE-FREE ZONE}

It is important not to forget the less specialised training essential for employees in all workplaces, such as courses in fire safety awareness. dbg recognises that the safety of patients and staff is paramount in dental practices, and accordingly offers a Basic Fire Safety Awareness \&t Marshal training course for staff.

Under the Regulatory Reform (Fire Safety) Order 2005, all dental staff must have specific training on what to do in the event of a fire in their practice. The dbg programme is specifically tailored to dental practices, and gives staff three hours of verifiable CPD whilst teaching them how to: understand the causes of fire; recognise fire hazards and take precautions against them; raise the alarm; identify escape routes; classify different categories of fire; use fire extinguishers of varying types correctly; and understand the role of the Fire Marshal.

Contact dbg today to ensure that your practice is protected against the threat of fire.

Reader response number 57

\section{REFURBISHMENT SPECIALISTS}

$\mathrm{C}$ and S ltd offer services ranging from the fit-out of a new property or refurbishment of an existing practice to small alterations, or the upgrade of a specific area. Their refurbishment programme helps guide you through every stage so that you stay in control every step of the way.

For a free, no obligation assessment of your project requirements, contact C and S ltd for a fast, detailed estimate. Reader response number 58 